\title{
Role of Nonenhanced Spiral Computed Tomography Scan in Refractory Irritative Bladder Symptoms
}

\author{
Hamed Akhavizadegan ${ }^{1, *}$ \\ ${ }^{1}$ Assistant Professor in Urology, Baharloo Hospital, Tehran University of Medical Sciences, Tehran, Iran \\ "Corresponding author: Hamed Akhavizadegan, M D, Urology Clinic, Baharloo Hospital, Behdari St, RahAhan Sq, Tehran, Iran. Tel: +98-2155669300, Fax: +98-2155648189, E-mail: \\ h-akhavizadegan@tums.ac.ir
}

Received 2016 December 08; Revised 2017 February 06; Accepted 2017 February 25.

\begin{abstract}
Introduction: In this study, we presented two cases with lower urinary tract symptoms and missed urinary stone that were diagnosed by the computed tomography (CT) scan.

Case Presentation: We reported two elderly men with severe irritative bladder symptoms who failed to be diagnosed with routine work-ups, including urine analysis and culture, urine cytology, ultrasonography, cystoscopy and urodynamic studies. Abdominopelvic nonenhanced spiral CT scan was performed. Missing stones were detected by the helical CT scan without contrast, which were treated by intracorporal lithotripsy. The symptoms were relieved following stone removal.

Conclusions: According to these two cases, there may be a diagnostic role for CT scan in evaluation of the irritative bladder symptoms.
\end{abstract}

Keywords: Voiding Dysfunction, Irritative Bladder Symptoms, CT Scan, Stone, LUTS

\section{Introduction}

Voiding dysfunction, even in mild to moderate cases, can remarkably impair quality of life in patients (1). Although there are numerous effective medications to alleviate these symptoms, it is recommended to perform full work-ups to rule out any important etiologies. This approach helps avoiding unnecessary medications and interventions. There are step by step guidelines for diagnosis and treatment of these symptoms (2). Origins of lower urinary tract symptoms can be urethra, prostate, bladder and uretero-vesical junction (UVJ). The implemented approach should differentiate symptoms attributable to these four anatomic regions. In addition to the history and physical examination, urine analysis and culture, analysis and culture of the first urine drops, a four-glass study, urine cytology, cystourethroscopy, ultrasonography, and urodynamics are also helpful. In this report, the use of nonenhanced spiral abdominopelvic CT scan is demonstrated to be a useful imaging modality in some cases.

\section{Case Presentation}

A sixty-year-old man presented to our hospital with refractory irritative bladder symptoms. Before the current visit, he had severe lower urinary tract symptoms refractory to alpha blockers, followed by the transurethral resection of prostate (TURP) three months before. Nevertheless, his symptoms were not relieved following TURP. Urine analysis and culture and ultrasound examination were normal. The cystoscopy under local anesthesia was also normal other than a small residual median lobe. The urodynamic study revealed detrusor overactivity with spontaneous contractions with no evidence of outlet obstruction. The patient did not respond to increasing dosage of anticholinergics. In another TURP attempt, the residual median lobe was resected, but it did not relieve the symptoms. Spiral CT scan of the abdomen and pelvis without oral and intravenous contrast media was requested. Surprisingly, a left ureterovesical junction stone without obstruction and with a normal upper tract was seen (Figure1). After transureteral lithotripsy (TUL), the patient was cured completely.

The second case was a 72-year-old man with a history of open prostatectomy 12 years ago, complaining of lower urinary tract symptoms. He had sustained a work-up similar to the first case without any findings. The CT scan revealed a small missed bladder stone, which its extraction eliminated the patient's symptoms.

\section{Discussion}

There are two types of lower urinary tract symptoms: obstructive or voiding phase symptoms and irritative or storage phase symptoms. Obstructive bladder symptoms include poor stream, low urine force, intermittency, hesitancy and straining. Irritative bladder symptoms contain 


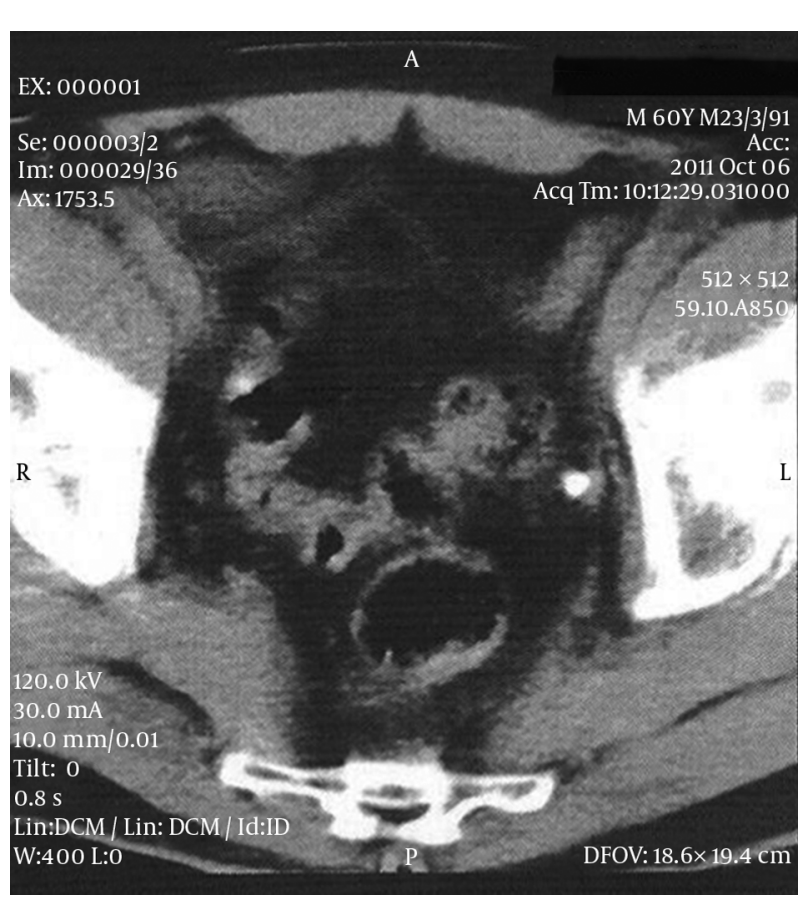

Figure 1. Abdominopelvic Nonenhanced Helical Computed Tomography Scan in the Patient

urgency, frequency, urge incontinence, nocturia and dysuria. Overactive bladder manifests with irritative bladder symptoms. Only the urgency is the hallmark symptom of bladder hyperactivity.

Irritative bladder symptoms can be idiopathic and without any obvious etiology or secondary with a specific pathology. It is very important to find the main cause of the symptoms, otherwise unnecessary interventions or longterm medical therapy will be offered to the patients. Unfortunately, secondary types are commonly resistant to these treatments.

Irritative and obstructive are misleading words. Irritative symptoms can be produced not only by irritative etiologies such as urethritis or prostatitis, but also by obstructive causes such as benign prostatic hyperplasia and urethral stricture. According to this paradox, nowadays, voiding phase and storage phase symptoms are replaced successfully.

Lower irritative urinary tract symptoms are usually produced by prostate, bladder, urethra or distal ureter pathologies. Each of these organs can be evaluated by different modalities.

Urethra can be checked by microscopic evaluation and culture of the first urine drops, urethral swab direct smear and culture and finally urethroscopy.

Prostate can be evaluated by the four-glass study, tran- srectal ultrasonography and cystoscopy. Bladder survey includes urine analysis and culture, ultrasonography, urine cytology and cystoscopy. Evaluation of ureterovesical junctions as part of trigone can be performed by ultrasound examination with full bladder.

A urodynamic study by recording the changes in bladder, urethra, volume, flow and pressure plays an important role in this diagnostic dilemma.

Even after localizing the symptoms to specific organ, there are many different pathologies that can cause these symptoms. For instance, the vesical part of these etiologies can be myogenic, neurogenic or idiopathic. Myogenic and neurogenic types should be managed according to their main pathology. After the complete work-up to rule out the detectable etiologies, symptoms can be alleviated by certain medication, such as anticholinergics effectively.

Regarding the work-up, urine analysis and culture, ultrasound scan, cystoscopy and urodynamics can reveal the main problem in most of the cases (Figure 2).

Ultrasound examination, which plays a great role in this diagnostic approach, is highly operator-dependent(3). The main advantage of ultrasound examination in the era of LUTS is detection of prostate, bladder and UVJ problems.

In these two patients, pathologies in bladder and UVJ were missed.

In first case, the UVJ stone was missed. As mentioned above, the only routine modality used to evaluate UVJs is ultrasound examination. In the second case, a small bladder stone was missed. Although ultrasonography and cystoscopy had been performed in both patients, the main diagnosis was missed.

Bladder and UVJ ultrasound accuracy is mainly dependent upon an adequate bladder volume. Thus, the ability of the patient to hold more than $100 \mathrm{cc}$ urine is very important for proper investigation (4). These two cases could not tolerate a full bladder for ultrasonography.

Cystoscopy with local anesthesia, in a man with severe LUTS is difficult to tolerate. Thus, the bladder stone was missed too.

A nonobstructing old ureterovesical junction stone or missed small bladder stone in empty bladder, without any other finding, can cause a diagnostic dilemma in these cases. The nonenhanced helical CT scan as the best modality to localize a stone in a urinary system (5) can be helpful in similar cases.

\subsection{Conclusions}

In this report, we presented two patients with missed urolithiasis presenting with LUTS, who were treated effectively after a correct diagnosis through CT scan. In cases with LUTS that ultrasound examination has not 


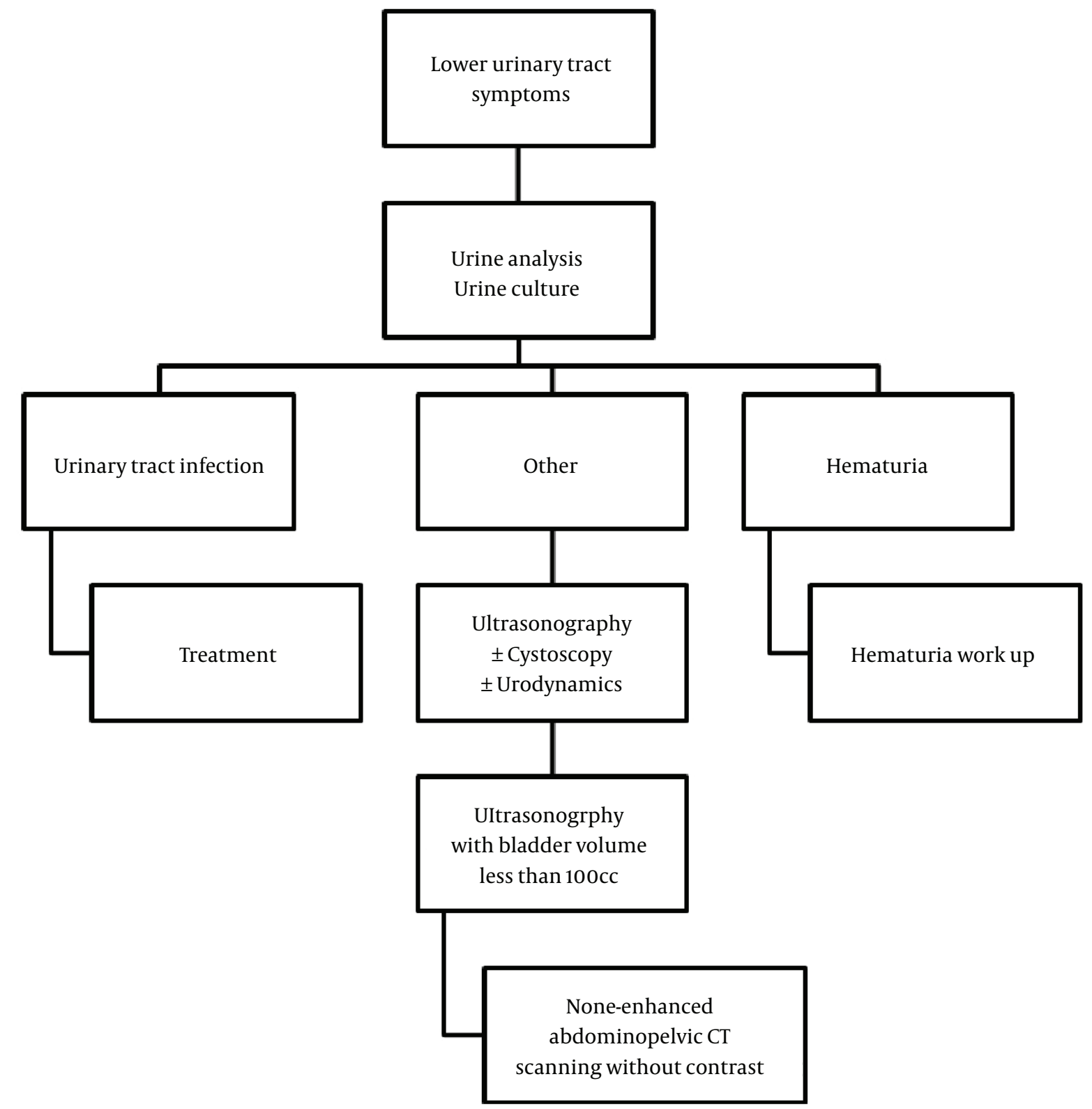

Figure 2. The Suggested Diagnostic Approach for Irritative Bladder Symptoms

been performed with adequate bladder volume, the spiral nonenhanced abdominopelvic CT scan and cystoscopy with general anesthesia is highly recommended to assess the ureterovesical junctions and bladder more conveniently and prevent unnecessary invasive procedures.

\section{References}

1. Coyne KS, Sexton CC, Thompson CL, Clemens JQ, Chen CI, Bavendam T, et al. Impact of overactive bladder on work productivity. Urology. 2012;80(1):97-103. doi: 10.1016/j.urology.2012.03.039. [PubMed: 22748868].

\section{Footnote}

Conflict of Interests: The authors declare that they have no conflict of interest. 
2. Murphy AM, Krlin RM, Goldman HB. Treatment of overactive bladder: what is on the horizon?. Int Urogynecol J. 2013;24(1):5-13. doi: 10.1007/s00192-012-1860-6. [PubMed: 22752012].

3. Pavlica P, Gaudiano C, Barozzi L. Sonography of the bladder. World J Urol. 2004;22(5):328-34. doi: 10.1007/s00345-004-0415-y. [PubMed: 15290205].

4. Moesbergen TC, de Ryke RJ, Dunbar S, Wells JE, Anderson NG. Dis- tal ureteral calculi: US follow-up. Radiology. 2011;260(2):575-80. doi: 10.1148/radiol.11101077. [PubMed: 21555351].

5. Semins MJ, Feng Z, Trock B, Bohlman M, Hosek W, Matlaga BR. Evaluation of acute renal colic: a comparison of non-contrast CT versus 3T non-contrast HASTE MR urography. Urolithiasis. 2013;41(1):43-6. doi: 10.1007/s00240-012-0525-8. [PubMed: 23532422]. 